\title{
Regime Monetário de Meta de Inflação em um Ambiente de Heterogeneidade de Estratégias de Formação de Expectativas de Inflação*
}

\author{
Jaylson Jair da Silveira \\ Professor - Departamento de Economia e Relações Internacionais (UFSC) \\ Endereço para contato: Campus Universitário, Trindade - Florianópolis -SC \\ CEP: 88040-970 - E-mail: jaylson@cse.ufsc.br \\ Gilberto Tadeu Lima \\ Professor - Faculdade de Economia, Administração e Contabilidade (FEA/USP) \\ Endereço para contato: Av. Prof. Luciano Gualberto, 908 - Cidade Universitária - São Paulo \\ CEP: 05508-010 - E-mail: giltadeu@usp.br
}

Recebido em 14 de agosto de 2012. Aceito em 14 de fevereiro de 2013.

\begin{abstract}
Resumo
Estudamos a dinâmica de um regime monetário de meta de inflação em um contexto macroeconômico representado por um modelo de três equações (curva IS, curva de Phillips e regra de juros) e com heterogeneidade de estratégias de formação de expectativas de inflação. Os agentes econômicos escolhem entre usar a meta de inflação anunciada como previsor da inflação futura (estratégia crédula) ou pagar um custo aleatório (e desconhecido a priori) para prevê-la com perfeição (estratégia cética). A distribuição dessas estratégias na população de agentes segue uma dinâmica evolucionária com e sem mutação endógena. Concluímos que a credibilidade plena não é uma condição necessária para o alcance da meta de inflação, dado que a heterogeneidade de expectativas de inflação, na presença de mutação e com alcance da meta de inflação, é um equilíbrio evolucionário.
\end{abstract}

\section{Palavras-Chave}

meta de inflação, expectativa de inflação, dinâmica evolucionária

\begin{abstract}
We study the dynamics of an inflation targeting regime in a macroeconomic context represented by a three-equation model (IS curve, Phillips curve and interest rate rule) and with heterogeneous strategies to form expectations of inflation. Economic agents choose between using the official inflation target as predictor of future inflation (credulous strategy) or paying a random cost (unknown a priori) to perfectly predict it (skeptical strategy). The distribution of these strategies in the population follows an evolutionary dynamics with and without endogenous mutation. We find that full credibility is not
\end{abstract}

\footnotetext{
- Os autores agradecem auxílios de pesquisa fornecidos pelo CNPq, bem como os comentários valiosos dos pareceristas anônimos, que em muito contribuíram para a melhoria do artigo. Naturalmente, os erros remanescentes são de nossa exclusiva responsabilidade.
} 
a necessary condition for reaching the inflation target, given that heterogeneity of inflation expectations, when there is mutation, is an evolutionary equilibrium but the inflation target is nonetheless reached.

\section{Keywords}

inflation target, inflation expectation, evolutionary dynamics

\section{JEL Classification}

C73, E31, E52

\section{Introdução}

Uma adequada administração e ancoragem das expectativas dos agentes privados, formadores de preços ou não, em relação à inflação futura é uma dimensão essencial do regime monetário de meta de inflação. Por sua natureza de indicador antecedente de riscos inflacionários futuros, tal como percebidos e estimados pelos agentes econômicos privados, essas expectativas fornecem informações fundamentais para a condução da política monetária. Na prática, especialmente em um regime monetário de meta de inflação, a expectativa de inflação dos agentes privados é uma variável que assume, seja implícita ou explicitamente, o papel de meta intermediária da política monetária. ${ }^{1}$

Por sua vez, as expectativas de inflação dos agentes privados são heterogêneas em uma dupla dimensão: não somente as expectativas individuais em si são diversas, mas, inclusive, e mesmo mais relevante do ponto de vista da necessidade de administração dessas expectativas por parte da autoridade monetária, também o são os mecanismos utilizados na sua formação. De fato, como devidamente reportado na seção seguinte, existem evidências empíricas robustas, obtidas através tanto de pesquisas de opinião como de experimentos de laboratório, não apenas de que essas duas dimensões da heteroge-

1 O Banco Central do Brasil, para citar um exemplo próximo, inclui as expectativas de inflação que mede junto a agentes privados entre os seis componentes em que decompõe a variação do IPCA. Em 2011, por exemplo, a maior contribuição individual para a variação absoluta (de 6 p.p.) desse índice adveio dos preços livres (2,85 p.p.), seguida da contribuição dos preços administrados (1,64 p.p.) e dos choque de oferta (0,94 p.p.). Por sua vez, a inércia e as expectativas contribuíram com 0,78 p.p. e 0,51 p.p, ou seja, 12,0\% e 7,8\% da variação total do IPCA, respectivamente. Por fim, o câmbio apresentou contribuição negativa $(-0,22$ p.p.)

(Banco Central do Brasil, 2012). 
neidade de expectativas de inflação dos agentes privados são significativas, mas, inclusive, de que essa heterogeneidade é persistente. E, dado o propósito do presente artigo, adquire saliência especial a evidência empírica robusta de que a distribuição de estratégias de formação de expectativas de inflação entre os agentes privados varia endogenamente ao longo do tempo.

Entretanto, a grande maioria da literatura sobre meta de inflação e política monetária (de taxa de juros) ótima ainda está baseada na hipótese de homogeneidade de expectativas de inflação dos agentes privados, tipicamente, ainda que não exclusivamente, assumindose que essas expectativas são formadas de acordo com algum dos sentidos do conceito de expectativa racional. Por outro lado, poucas contribuições recentes têm explorado, tanto teórica como empiricamente, implicações da heterogeneidade de mecanismos de formação de expectativas de inflação em termos de política monetária ótima em um regime de meta de inflação. A seção seguinte não somente resenha sinteticamente uma amostra representativa dessas contribuições, mas, o que é mais importante, detalha, nesse contexto, a inovação e a correspondente contribuição do presente artigo.

Colocando antecipadamente, a contribuição do presente artigo para a literatura sobre o tema advém do estudo da dinâmica de um regime monetário de meta de inflação em um modelo de três equações e com heterogeneidade de estratégias de formação de expectativas de inflação. Os agentes econômicos podem optar por usar a meta de inflação anunciada como previsor da inflação futura (estratégia crédula) ou pagar um custo aleatório (e desconhecido a priori) para prever com perfeição esta inflação (estratégia cética). A evolução da distribuição de estratégias de formação de expectativas de inflação é concebida como um processo evolucionário, composto por duas forças: seleção e mutação. Enquanto a primeira reflete a atratividade relativa das estratégias em termos de payoffs, a segunda reflete escolhas aleatórias desconectadas dos payoffs.

A dinâmica de coevolução da macroeconomia e da distribuição das estratégias de formação de expectativas apresenta um único equilíbrio, caracterizado por credibilidade incompleta, se há perturbação advinda da mutação endógena, e credibilidade plena, no caso contrário. Porém, nos dois casos o produto converge para o seu nível natural, as taxas de inflação esperada e observada tornam-se iguais 
à meta preestabelecida e a taxa nominal de juros acaba ancorada na meta de inflação e na taxa de juros real natural.

A sequência deste artigo está assim organizada. A próxima seção resenha brevemente a literatura relacionada e, com isso, contextualiza as contribuições do presente artigo. A seção 3, por sua vez, desenvolve um modelo dinâmico do qual resultam as contribuições do artigo, enquanto a seção 4 sintetiza essas contribuições.

\section{Literatura Relacionada e Contribuições deste Artigo}

A formulação e execução da política monetária em um regime de meta de inflação requer uma inferência acerca da forma com que o público forma expectativas de inflação. Muito embora seja recorrente na literatura correspondente o recurso a alguma variante da hipótese de expectativa racional, alguns autores reconhecem que uma modalidade de incerteza com que inevitavelmente se defrontam os gestores de política monetária diz respeito à maneira pela qual os agentes privados originalmente formam e posteriormente revisam suas expectativas. Orphanides e Williams (2007, p. 1407), por exemplo, assim se manifestam:

There is a growing literature that analyzes a variety of alternative models of expectations formation. The key conclusion we take from our reading of this literature is that there is a great deal of uncertainty regarding exactly how private expectations are formed. In particular, the standard assumption of rational expectations may be overly restrictive for monetary policy analysis, especially in the context of an economy undergoing structural change. But, the available evidence does not yet provide unequivocal support for any other single model of expectations formation. Therefore, fundamental uncertainty about the nature of expectations formation appears to be an unavoidable aspect of the policy environment central banks face today. 


\subsection{Evidências Empíricas sobre Formação de Expectativas de Inflação}

As evidências empíricas disponíveis sobre formação de expectativas de inflação podem ser divididas em evidências obtidas através de pesquisa de opinião e evidências geradas por experimentos controlados de laboratório. Dentre estes últimos, uma amostra representativa inclui os estudos de Hommes et al. (2005), Adam (2007) e Pfajfar e Zakelj (2010), nos quais foram encontradas evidências robustas de formação de expectativas individuais com base em alguma modalidade de racionalidade limitada. ${ }^{2}$

Em estudo recente, Assenza et al. (2011) utilizam experimentos de laboratório para investigar o processo de formação de expectativas de agentes econômicos dentro de um arcabouço Novo-Keynesiano. Os participantes preveem a taxa de inflação em três cenários diferentes em termos de suposição acerca das expectativas de hiato de produto, previsão perfeita, previsão ingênua (espera-se a repetição do valor no período anterior) e previsões de grupos de indivíduos (os agentes econômicos são divididos aleatoriamente em dois grupos, um deles formando expectativas sobre a taxa de inflação, o outro formando expectativas sobre o hiato de produto). Neste último cenário, em todos os tratamentos, o regressor significante mais popular foi o último valor observado da variável a ser prevista, seguido, na maioria dos tratamentos, pela previsão própria mais recente ou pelo penúltimo valor observado da variável a ser prevista. De maneira geral, os autores encontram evidências de que (1) os indivíduos baseiam suas predições predominantemente em observações passadas, seguindo, para tanto, heurísticas de previsão simples e (2) o aprendizado individual assume a forma de mudança de uma heurística para outra.

Em termos de estudos com dados de pesquisa de opinião, menção inicial merece ser feita a Carroll (2003), no qual são encontradas evidências, a partir de dados para a economia norte-americana, de que (1) as expectativas de inflação tanto de consumidores como economistas profissionais são consideravelmente heterogêneas e (2) a distribuição dessa heterogeneidade varia ao longo do tempo em resposta a mudanças nas condições econômicas. Evidências empíricas semelhantes são obtidas em Mankiw, Reis e Wolfers (2004), também a partir de dados para a economia norte-americana.

2 Resenhas dessa literatura de economia experimental são feitas em Duffy (2008) e Hommes (2011) 
Além disso, esses autores encontram evidências de formação de expectativas individuais com base em alguma modalidade de racionalidade limitada. Branch $(2004,2007)$, por sua vez, igualmente utilizando dados norte-americanos, encontra evidências de que (1) as expectativas dos consumidores se distribuem entre racionais e adaptativas e (2) essa distribuição varia ao longo do tempo em resposta a erros de previsão passados.

Capistrán e Timmermann (2009) detectam que a heterogeneidade de expectativas de inflação de profissionais de previsão varia ao longo do tempo conforme o comportamento da inflação corrente. Pfajfar e Santoro (2010), por sua vez, igualmente utilizando dados para a economia norte-americana, encontram evidências de que a heterogeneidade de expectativas de inflação é persistente e identificam três mecanismos diferentes de formação de expectativas, a saber, estático ou autorregressivo, quase plenamente racional e de aprendizado adaptativo com informação rígida. Por outro lado, Weber (2010) investiga o desempenho de diferentes regras de aprendizado na explicação de dados de pesquisa de opinião sobre expectativas de inflação de unidades familiares e profissionais de previsão em cinco economias europeias (França, Alemanha, Itália, Holanda e Espanha). O autor detecta que as expectativas de inflação desses dois grupos são bastante heterogêneas intra e entre si, sendo que os erros de previsão quadráticos médios são maiores para as unidades familiares que para os profissionais de previsão. Além disso, Weber (2010) encontra pouca evidência, para ambos os grupos de agentes, de que as expectativas de inflação são formadas de maneira racional.

\subsection{Modelos de Meta de Inflação com Heterogeneidade de Expectati- vas de Inflação}

Como antecipado na introdução, algumas contribuições relativamente recentes têm explorado, tanto teórica como empiricamente, implicações da heterogeneidade de mecanismos de formação de expectativas de inflação no que diz respeito à política monetária ótima em um regime de meta de inflação. No que segue, resenhamos sinteticamente uma amostra representativa dessas contribuições com o intuito de contextualizar tanto a inovação como a correspondente contribuição do presente artigo. 
Três origens diferentes dessa heterogeneidade têm sido tradicionalmente exploradas na literatura em questão. Especificamente falando, previsões heterogêneas de inflação podem ter origem no fato de os agentes econômicos (i) empregarem diferentes modelos, (ii) basearem-se em conjuntos informacionais diversos e (iii) serem dotados de diferentes níveis de capacidade de processamento da informação. Enquanto algumas teorias postulam a existência de fricções informacionais que geram rigidez expectacional, outras conjecturam que os agentes podem agir como econometristas quando realizam previsões. Esta última abordagem, conhecida como aprendizado adaptativo, é extensivamente apresentada e discutida em Evans e Honkapohja (2001). Em termos da abordagem da informação rígida, por sua vez, várias contribuições (e.g. Carroll, 2006; Mankiw e Reis, 2002) mostram como é possível conceber regras de estabelecimento de preços dependentes do estado sob as quais as expectativas são atualizadas somente em intervalos fixos.

Mankiw e Reis (2002) desenvolvem um modelo dinâmico de ajustamento de preços baseado no suposto de que a informação não se dissemina instantaneamente na população de agentes. Embora os agentes sejam racionais, a existência de custos de aquisição de informação ou de (re)otimização faz com que a difusão de informações a respeito das condições macroeconômicas seja lenta. Na presença de custos dessa natureza, os preços, embora estejam sempre variando, nem sempre são estabelecidos com base em todas as informações existentes. Daí, portanto, rotularem sua contribuição de modelo de informação rígida e não de modelo de preço rígido. Os autores supõem que, a cada período, uma fração da população atualiza seu conjunto de informações sobre o estado corrente da economia e calcula preços ótimos com base nesse conjunto atualizado. $\mathrm{O}$ restante da população, por sua vez, continua a estabelecer preços com base em um conjunto de informações desatualizado.

Carroll (2006) propõe uma interessante abordagem da formação de expectativas, baseada na epidemiologia, na qual somente um pequeno conjunto de agentes (previsores profissionais plenamente racionais) formula suas próprias expectativas, as quais então se espalham na população através dos veículos de notícias. Porém, nem todos os demais agentes dedicam atenção constante e cuidadosa ao noticiário macroeconômico. $\mathrm{O}$ autor supõe que esses agentes absorvem o conteúdo econômico das notícias de forma probabilística, de uma manei- 
ra análoga à difusão de uma doença na população. Logo, leva algum tempo para que notícias de mudanças nas condições macroeconômicas venham a ser absorvidas pelos demais agentes. Carroll (2006) mostra que esse modelo tem um bom desempenho empírico na explicação da dinâmica das expectativas de inflação e desemprego. Segundo ele, enquanto Mankiw e Reis (2002) não fornecem microfundamentos explícitos para seu suposto de custos informacionais, seu modelo fornece uma microfundamentação explícita, baseada em modelos epidemiológicos, para uma equação expectacional agregada.

Outra contribuição interessante nessa linha mais recente de modelos de imperfeição informacional foi desenvolvida por Woodford (2003), que se baseia no suposto de que o agente tem uma capacidade limitada de absorção de informação. Posto que os formadores de preços aprendem sobre a política monetária através desse canal de informação limitada, é como se observassem a política monetária com um erro aleatório e, assim, tivessem que resolver um problema de extração de sinal. Portanto, uma diferença básica entre as contribuições de Mankiw e Reis (2002) e de Woodford (2003) diz respeito à maneira pela qual a informação chega aos agentes. Enquanto nesta última os formadores de preço recebem a cada período um sinal com ruído sobre a política monetária, na primeira os formadores de preços adquirem informação perfeita sobre a política monetária em um dado período com certa probabilidade.

Por outro lado, quando é suposto que as expectativas de inflação do setor privado são formadas de acordo com a abordagem do aprendizado adaptativo, isso significa supor que os agentes não conhecem o processo exato seguido pelas variáveis endógenas, porém estimam recursivamente uma lei de movimento consistente com aquela que a autoridade monetária implementaria sob expectativas racionais. A literatura moderna sobre esse tópico foi iniciada por Marcet e Sargent (1989), os primeiros autores a aplicar técnicas de aproximação estocástica ao estudo de convergência de algoritmos de aprendizado.

Da perspectiva evolucionária na qual se baseia o presente artigo, parece inadequado supor um comportamento de racionalidade limitada no que se refere à fixação de preço que se caracteriza pela sujeição a uma regra mecânica de atualização do conjunto de informações, como é o caso do modelo de Mankiw e Reis (2002), no qual é adotada uma especificação à la Calvo (1983), ou seja, o momento 
de reajuste do preço é aleatório e, portanto, não é uma variável de decisão da firma. No modelo desenvolvido na seção seguinte, por sua vez, a decisão de revisão da estratégia de formação de expectativas de inflação é tomada continuamente com base em considerações de benefícios líquidos (de custo) esperados. A abordagem do aprendizado adaptativo, por sua vez, conforme descrita sucintamente em parágrafos anteriores, claramente atribuiu ao agente econômico uma capacidade cognitiva que este, no mundo real, normalmente não demonstra possuir, tal como comprovado pela evidência empírica reportada na subseção anterior.

Por outro lado, Diron e Mojon (2005) representam uma interessante contribuição com a qual está alinhada, mutatis mutandis, a dinâmica evolucionária de revisão da estratégia de formação de expectativa de inflação contida no modelo elaborado na seção seguinte. Afinal, os autores mostram que o erro de previsão incorrido quando se assume que inflação futura será igual à meta de inflação anunciada pela autoridade monetária é tipicamente pelo menos tão pequeno quanto (e mesmo frequentemente menor que) os erros de previsão associados ao uso dos principais modelos de previsão disponíveis. A seu ver, e certamente ao nosso, esse resultado não surpreende: afinal, em teoria, a maior virtude do anúncio crível de uma meta de inflação expressa em termos quantitativos é precisamente a de ancorar as expectativas de inflação. Essa ancoragem, por sua vez, auxilia a autoridade monetária em sua tentativa de estabilizar a inflação ao nível pré-anunciado.

Em seu estudo empírico, Diron e Mojon (2005) utilizam dados de 1995 a 2004 para um conjunto de sete países desenvolvidos e da zona do Euro que adotam o regime de meta de inflação. Em todos eles, a previsão de que a inflação seria igual à meta anunciada implicou um erro de previsão menor que as previsões derivadas de modelos de inflação do tipo passeio aleatório ou AR (2), modelos que eles consideram representativos dos principais modelos de previsão baseados em representações econométricas. De fato, a previsão de que a inflação seria igual à meta anunciada bateu inclusive a chamada previsão Consensus, obtida a partir das previsões de economistas profissionais de atuação mundial, para a zona do Euro, o Canadá, a Suécia e a Suíça, além de ter ido tão bem quanto a previsão Consensus para o Reino Unido. Além desses estudos empíricos, os autores utilizam um modelo macroeconômico de três equações 
(curva IS, curva de Phillips e regra de juros) semelhante ao que será elaborado na seção seguinte, a partir do qual concluem que o desempenho macroeconômico, sob vários aspectos, será tanto melhor quanto maior for a fração de agentes econômicos que utilizam a meta de inflação como preditor da inflação futura. Ao contrário do modelo a ser elaborado na seção seguinte, porém, a fração de agentes econômicos que assim procedem é uma constante exogenamente determinada, e não uma variável endógena sujeita a uma dinâmica evolucionária com e sem mutação endógena.

Na mesma linha de Diron e Mojon (2005), embora de uma perspectiva principalmente teórico-formal, Kapadia (2005) sustenta que é plausível supor que, em países com um regime de meta de inflação crível, algumas firmas podem simplesmente considerar que o melhor preditor da inflação futura é a meta de inflação anunciada pela autoridade monetária. Com base na elaboração de um modelo macroeconômico de três equações bem mais completo e devidamente microfundamentado que o utilizado por Diron e Mojon (2005), Kapadia (2005) igualmente conclui que o desempenho macroeconômico, sob vários aspectos, será tanto melhor quanto maior for a fração de firmas que utilizam a meta de inflação como preditor da inflação futura. Mas, ao contrário do modelo a ser elaborado na seção seguinte, porém, essa fração é uma constante exogenamente determinada e não uma variável sujeita a uma dinâmica endogenamente determinada.

Brazier et al. (2008), por sua vez, desenvolvem um modelo de gerações sobrepostas no qual os agentes precisam prever a inflação, e o fazem utilizando duas regras de bolso ou heurísticas, a saber, uma que prescreve prever a inflação com base na inflação passada, outra que prescreve utilizar a meta de inflação anunciada pela autoridade monetária para realizar essa previsão. Uma implicação do modelo é que a inflação tende ser tanto menos variável e persistente quanto maior for a proporção de agentes que utilizam a meta de inflação como preditor da inflação futura. Ao contrário de Diron e Mojon (2005) e Kapadia (2005), por sua vez, os autores utilizam um modelo de mudança de heurística para contrastar o desempenho de diferentes regras de política monetária. Esse modelo, entretanto, embora seja baseado no desempenho passado relativo das várias heurísticas, como é o caso do modelo evolucionário de revisão de estratégia de formação de expectativa de inflação a ser elaborado na seção se- 
guinte, é consideravelmente diferente deste. De fato, o modelo de mudança de heurística utilizado pelos autores é baseado na contribuição de Brock e Hommes (1997), os quais, por sua vez, embasam seu modelo de tomada de decisão nos modelos de escolha discreta do tipo logit multinomial desenvolvidos em Manski e McFadden (1981).

De Grauwe (2010), por sua vez, igualmente desenvolve um modelo no qual os agentes têm limitações cognitivas e, em consequência, utilizam heurísticas simples, porém viesadas, para prever a inflação futura. Assim como Brazier et al. (2008), o autor supõe que os agentes utilizam duas regras de bolso ou heurísticas para prever a inflação futura (uma extrapolativa, outra baseada no uso da meta de inflação), além de igualmente basear sua análise da dinâmica de mudança de heurística na contribuição de Brock e Hommes (1997). Na mesma linha, Branch e McGough (2010) estendem sua contribuição anterior (Branch e McGough, 2009), na qual a distribuição de expectativas heterogêneas é uma constante exogenamente determinada. Para tanto, supõem uma dinâmica endógena para a distribuição de expectativas heterogêneas também baseada no arcabouço de mudança de heurística desenvolvido na contribuição de Brock e Hommes (1997).

\section{Um Modelo Macroeconômico de três Equações com Expecta- tivas Heterogêneas}

O cenário macroeconômico de referência, no qual a análise a ser desenvolvida no presente artigo será realizada, é representado pelo seguinte modelo macroeconômico de três equações:

$$
\begin{aligned}
& y_{t}-y_{n}=-\alpha\left(i_{t-1}-\pi_{t}^{e}-r_{n}\right), \\
& \pi_{t}=\pi_{t}^{e}+\beta\left(y_{t}-y_{n}\right), \\
& i_{t-1}=\pi_{t}^{e}+r_{n}+\gamma\left(\pi_{t-1}-\pi^{T}\right) .
\end{aligned}
$$

A primeira equação representa a curva IS em termos do desvio do produto $y_{t}$ no período $t$ com relação ao produto natural $y_{n}$. Sejam $\alpha>0$ uma constante paramétrica, $i_{t-1}$ a taxa nominal de juros no período $t-1, \pi_{t}^{e}=E_{t-1}\left(\pi_{t}\right)$ a inflação esperada em $t-1$ que vigorará 
em $t$ e $r_{n}$ a taxa de juros real natural. Se a taxa real de juros esperada no período $t-1$ para viger em $t$, dada pela diferença $i_{t-1}-\pi_{t}^{e}$, superar a (for superado pela) taxa natural de juros, o produto no período seguinte ficará abaixo (acima) do seu nível natural. A segunda equação é a curva de Phillips expandida pelas expectativas, sendo $\pi_{t}$ a taxa de inflação observada em $t$ e $\beta>0$ uma constante paramétrica. Finalmente, a terceira equação representa uma regra de juros, sendo $\gamma>0$ uma constante paramétrica e $\pi^{T}$ a meta da taxa de inflação anunciada pela autoridade monetária.

Posto que a principal contribuição pretendida por este artigo reside na incorporação de uma heterogeneidade na formação de expectativas de inflação que segue uma dinâmica evolucionária com e sem mutação endógena (motivada pela evidência empírica robusta, reportada na seção 2.1, de que essa heterogeneidade, muito embora varie endogenamente no tempo, é persistente), optamos pela utilização de um modelo macroeconômico de três equações que abstrai de alguns elementos incorporados, em doses e combinações variadas, na literatura sobre meta de inflação e política monetária ótima. A curva IS, por exemplo, não incorpora seja o hiato de produto passado, cuja presença tipicamente é justificada com base na noção de formação de hábito de consumo, seja o hiato de produto esperado, resultante da derivação microfundamentada dessa curva a partir da equação de Euler. A curva de Phillips, por sua vez, não inclui a inflação passada. E, no caso da regra de juros, não estão incluídos o hiato de produto (corrente ou esperado) e a taxa de juros passada (ou seja, não há suavização de juros). Além disso, nenhuma dessas relações inclui um componente de choque aleatório. ${ }^{3}$

Inserindo (3) em (1) e a função resultante em (2), o sistema (1)-(3) pode ser sintetizado como segue:

$$
\pi_{t}=\pi_{t}^{e}-\alpha \beta \gamma\left(\pi_{t-1}-\pi^{T}\right)
$$

Portanto, além da meta de inflação fixada pela autoridade monetária e da taxa de inflação passada, a taxa de inflação no presente depende diretamente da inflação esperada. Em linha com

3 Várias especificações alternativas desse modelo macroeconômico de três equações, incluindo diferentes combinações dos elementos não incorporados ao modelo utilizado neste artigo, podem ser encontradas em Woodford (2003) e Galí (2008). 
contribuições resenhadas na seção anterior, mas se diferenciando de todas elas em termos da dinâmica evolucionária que guiará a distribuição das estratégias de formação de expectativas de inflação, supomos aqui que um regime de meta de inflação, entendido como o comprometimento da política monetária com a busca de inflação baixa e estável expresso no anúncio público e crível de uma meta de inflação, é um exemplo específico de como a autoridade monetária pode fornecer uma "âncora convencional" ao processo de formação de expectativas de inflação sob incerteza. ${ }^{4}$

Considerando que, em um dado período qualquer, as expectativas dos agentes possam ser heterogêneas, $\pi_{t}^{e}$ é uma variável agregada dessas expectativas. Mais precisamente, suporemos que em um período $t$ qualquer há uma fração $\lambda_{t} \in[0,1] \subset \mathbb{R}$ dos agentes, que pode variar de um período para outro, que confia plenamente na efetividade da meta de inflação anunciada pela autoridade monetária para aquele período. Chamaremos este tipo de agente de crédulo. A fração restante, $1-\lambda_{t}$, é formada pelos agentes que não confiam na efetividade da referida política, ou seja, são agentes do tipo cético, que formam suas expectativas de forma plenamente racional, pagando um custo para prever com perfeição a taxa de inflação. Assim, considerando que os agentes formam suas expectativas sobre a inflação em $t$ no final do período $t-1$, a expectativa da inflação em $t$ pode ser posta como uma média ponderada pela distribuição de expectativas no período anterior $\left(\lambda_{t-1}, 1-\lambda_{t-1}\right)$, ou seja:

$$
\pi_{t}^{e}=\lambda_{t-1} \pi^{T}+\left(1-\lambda_{t-1}\right) \pi_{t}
$$

Logo, se inserirmos (5) em (4), obteremos o desvio da inflação observada com relação à meta no período $t$ como uma função do respectivo desvio no período anterior e do grau de credibilidade da política monetária:

$$
\pi_{t}-\pi^{T}=\frac{-\alpha \beta \gamma}{\lambda_{t-1}}\left(\pi_{t-1}-\pi^{T}\right) \text {, para qualquer } \lambda_{t-1}>0 .
$$

4 Por exemplo, o estudo sobre a formação de expectativas de inflação no Brasil entre o início de 1999 e o início de 2005, elaborado por Cerisola e Gelos (2005), revelou que a meta de inflação foi um dos mais importantes determinantes da inflação esperada. O estudo empírico de Bevilaqua, Mesquita e Minella (2007), por seu turno, cobrindo o período entre o final de 2002 e o final de 2006, igualmente detectou que a meta de inflação desempenhou um papel fundamental como atrator para as expectativas de inflação. 
Cabe salientar que para um dado desvio passado da inflação com relação à meta, $\pi_{t-1}-\pi^{T}$, o respectivo desvio no presente, $\pi_{t}-\pi^{T}$, apresentará um sinal oposto e seu módulo será tanto menor quanto maior for o grau de credibilidade $\lambda_{t-1}$. Em outros termos, se a inflação observada em $t-1$ ficar acima da meta estabelecida pela autoridade monetária, a taxa nominal de juros neste período aumentará, afetando negativamente o produto no período seguinte, o qual ficará abaixo do seu nível natural. Este desaquecimento pressiona os preços para baixo em $t$. Isto é previsto pelos agentes céticos, que incorporam este efeito na sua previsão feita em $t-1$ da inflação em $t$. Quanto maior o grau de credibilidade da política de meta de inflação, ou seja, quanto maior a fração de agentes crédulos, menor será o efeito forward looking gerado pelos agentes céticos.

Passemos à análise do ajustamento do grau de credibilidade do regime monetário de meta de inflação, ou seja, da evolução da proporção de agentes crédulos na economia. Adotaremos a hipótese de que tais agentes podem incorrer em uma perda se a inflação observada se desviar da meta oficial de inflação utilizada como previsor da inflação. Mais precisamente, assumiremos que esta perda cresce com o quadrado do desvio da inflação observada com relação à meta:

$$
-\delta\left(\pi_{t}-\pi^{T}\right)^{2}
$$

sendo $\delta>0$ uma constante paramétrica.

Por sua vez, os agentes céticos não incorrem em perda por desviarem da inflação observada em cada período, pois, por hipótese, são capazes de prever com perfeição a inflação que vigorará no período. Entretanto, para estimar a inflação com perfeição cada agente cético arca com um custo, desconhecido a priori. Em outras palavras, suporemos que os agentes céticos em $t-1$ realizam uma subotimização, ou seja, inferem a inflação a ser observada em $t$ sem conhecerem com precisão os gastos necessários para tal inferência. Dessa forma, a perda efetiva do $i$-ésimo agente cético pode ser considerada uma variável aleatória $c_{i}$ com distribuição contínua de probabilidades $F: \mathbb{R}_{+} \rightarrow[0,1] \subset \mathbb{R}$.

Suporemos que os agentes avaliam suas estratégias de previsão da inflação a cada transição entre períodos consecutivos. Cada agente crédulo 
extrai informação unicamente da comparação de sua perda com a incorrida por outro agente escolhido aleatoriamente, segundo uma distribuição de probabilidades uniforme. A probabilidade desta comparação ser feita com um agente que escolheu a estratégia alternativa é igual a $1-\lambda_{t-1}$. Um agente crédulo em $t-1$ que compara, na transição do período $t-1$ para o período $t$, sua perda efetiva com a perda efetiva de um agente $i$ que tenha sido cético em $t-1$, passará a adotar a estratégia cética em $t$ se o custo incorrido em $t-1$ pelo agente cético $i$ tenha sido relativamente baixo, ou seja, se $c_{i}<\delta\left(\pi_{t-1}-\pi^{T}\right)^{2}$. Logo, a probabilidade de um agente crédulo em $t-1$, que extraiu informação de um agente cético na transição do período $t-1$ para o período $t$, tornar-se um agente cético em $t$ é dada por $\operatorname{Pr}\left(c_{i}<\delta\left(\pi_{t-1}-\pi^{T}\right)^{2}\right)=F\left(\delta\left(\pi_{t-1}-\pi^{T}\right)^{2}\right)$.

Se estes dois eventos forem estatisticamente independentes, o produto de suas probabilidades de ocorrência fornece a probabilidade de um agente crédulo em $t-1$ tornar-se um agente cético em $t$, dada por $\left(1-\lambda_{t-1}\right) F\left(\delta\left(\pi_{t-1}-\pi^{T}\right)^{2}\right)$. Como há $\lambda_{t-1}$ agentes céticos no período $t-1$, o valor esperado de agentes crédulos em $t-1$ que se tornam crédulos em $t$ é dada por:

$$
\lambda_{t-1}\left(1-\lambda_{t-1}\right) F\left(\delta\left(\pi_{t-1}-\pi^{T}\right)^{2}\right) .
$$

Por sua vez, dado que o custo incorrido pelos agentes crédulos em $t-1$, dado por $\delta\left(\pi_{t-1}-\pi^{T}\right)^{2}$, é publicamente observado na transição do período $t-1$ para o período $t$, e os agentes céticos conhecem seus custos de previsão incorridos em $t-1$ para prever a inflação em $t$, os agentes céticos em $t-1$ não precisam realizar comparações em pares das perdas para obterem informações sobre custos de previsão. Assim, o $i$-ésimo agente cético em $t-1$ passará a adotar a estratégia crédula no próximo período caso seu custo de previsão em $t-1$ tenha sido relativamente alto, ou seja, se $c_{i} \geq \delta\left(\pi_{t-1}-\pi^{T}\right)^{2}$. Logo, o $i$-ésimo agente cético em $t-1$ tornar-se-á um agente crédulo em $t$ com probabilidade $1-F\left(\delta\left(\pi_{t-1}-\pi^{T}\right)^{2}\right)$. Como a cada período todos os agentes céticos são potenciais revisores de estratégia, a fração esperada de agentes deste tipo em $t-1$ que passam a adotar a estratégia crédula em $t$ é simplesmente:

$$
\left(1-\lambda_{t-1}\right)\left[1-F\left(\delta\left(\pi_{t-1}-\pi^{T}\right)^{2}\right)\right]
$$


A diferença entre o influxo (9) e o efluxo (8) nos dá a taxa de variação da fração de agentes crédulos, $\lambda_{t}-\lambda_{t-1}$. Logo, a referida fração no período $t$ pode ser expressa como segue:

$$
\lambda_{t}=\lambda_{t-1}+\left(1-\lambda_{t-1}\right)\left[1-\left(1+\lambda_{t-1}\right) F\left(\delta\left(\pi_{t-1}-\pi^{T}\right)^{2}\right)\right] .
$$

Em um trabalho prévio (Silveira e Lima, 2008), e com outros objetivos, argumentamos que a concepção evolucionária do processo de escolha entre pagar ou não pagar o custo associado à previsão perfeita, aqui formalizada por (10), pode ser vista como uma estratégia de modelagem que contorna, por assim dizer, o problema de autorreferência associado ao cálculo otimizador, ou seja:

A razão é que conceber o processo de escolha entre pagar ou não pagar o custo associado à otimização como sendo ele próprio sujeito ao cálculo otimizador nos faria deparar com um problema de auto-referência ou regressão infinita (Conlisk, 1996). Afinal, para otimizar é necessário obter um conhecimento perfeito, o que tem custos. Sendo assim, a otimização correspondente não é ótima quando tal custo é ignorado. Eis a contradição: para não ignorá-lo é necessário incluir o custo de otimização na própria otimização, porém não é possível saber o custo da obtenção do conhecimento perfeito antes de conhecê-lo perfeitamente. (Silveira e Lima, 2008, p. 130)

Como em Lima e Silveira (2008), por sua vez, mas agora em um contexto diferente e com objetivos distintos, consideraremos que o processo evolucionário de escolha entre pagar ou não pagar o custo associado à previsão perfeita é influenciado por uma força adicional, além daquela de seleção captada por (10), a saber, uma força análoga à mutação em ambientes naturais. Como destacado por Samuelson (1997, cap. 7), enquanto o mecanismo de seleção busca captar o efeito direto dos payoffs sobre as escolhas de estratégias, a mutação é um resíduo composto por outros fatores que influenciam as escolhas de estratégias e que não foram incorporados no mecanismo de seleção. 
Em particular, no presente contexto a inclusão da mutação busca capturar choques, tais como: (1) mudanças institucionais exógenas, como troca do corpo administrativo da autoridade monetária ou outras alterações do processo de formulação de política monetária (que não acarrete, todavia, no abandono do regime monetário de meta de inflação) que geram perturbações exógenas no processo de formação de expectativas; (2) agentes econômicos que simplesmente experimentam uma nova estratégia; ou (3) entrada de novos agentes econômicos que, por inexperiência, decidem escolher aleatoriamente uma estratégia.

Como salienta Samuelson (1997, cap. 7), em um contexto econômico uma mutação pode ser interpretada como uma situação na qual um agente (o mutante) se abstém de comparar payoffs e escolhe sua estratégia de maneira aleatória. A importância relativa da força de mutação sobre um processo evolucionário em um ambiente econômico tende a aumentar se a diferença de payoffs entre estratégias diminui, pois os agentes tornam-se menos diligentes, pois tanto faz usar uma como a outra estratégia. No presente contexto a importância relativa da força de mutação tende a diminuir com o aumento do desvio da inflação observada com relação à meta, já que o custo incorrido pela credulidade aumenta. Sendo assim, suporemos que a medida de agentes mutantes que escolhem uma estratégia em um dado momento independentemente dos payoffs seja negativamente relacionada ao desvio da inflação observada com relação à meta, mais precisamente:

$$
\frac{\varepsilon \theta}{\varepsilon+\left(\pi_{t-1}-\pi^{T}\right)^{2}}
$$

na qual $\varepsilon>0$ e $0<\theta<1$ são constantes paramétricas. Assim, se a estratégia crédula tende a gerar perdas relativamente baixas, $\left(\pi_{t-1}-\pi^{T}\right)^{2} \rightarrow 0$, a fração de agentes mutantes tende a um valor máximo $\theta$. Todavia, se tal perda tende a se tornar muito grande, $\left(\pi_{t-1}-\pi^{T}\right)^{2} \rightarrow \infty$, a fração de mutantes tende a zero.

Do total de mutantes em (11), $\left\{\varepsilon \theta /\left[\varepsilon+\left(\pi_{t-1}-\pi^{T}\right)^{2}\right]\right\} \lambda_{t-1}$ são agentes crédulos e $\left\{\varepsilon \theta /\left[\varepsilon+\left(\pi_{t-1}-\pi^{T}\right)^{2}\right]\right\}\left(1-\lambda_{t-1}\right)$ são agentes céticos. Supondo que um agente mutante escolhe uma das duas estratégias de previsão com igual probabilidade, inferimos então 
que $\left\{\varepsilon \theta /\left[\varepsilon+\left(\pi_{t-1}-\pi^{T}\right)^{2}\right]\right\} \lambda_{t-1} / 2$ agentes crédulos mutantes e $\left\{\varepsilon \theta /\left[\varepsilon+\left(\pi_{t-1}-\pi^{T}\right)^{2}\right]\right\}\left(1-\lambda_{t-1}\right) / 2$ agentes céticos efetivarão mudanças de estratégia de $t-1$ para $t$. Logo, o fluxo líquido (positivo ou negativo) de agentes mutantes que passarão a adotar, em um dado período $t$, a estratégia crédula será:

$\left[\frac{\varepsilon \theta}{\varepsilon+\left(\pi_{t-1}-\pi^{T}\right)^{2}}\right]\left(1-\lambda_{t-1}\right) \frac{1}{2}-\left[\frac{\varepsilon \theta}{\varepsilon+\left(\pi_{t-1}-\pi^{T}\right)^{2}}\right] \lambda_{t-1} \frac{1}{2}=\left[\frac{\varepsilon \theta}{\varepsilon+\left(\pi_{t-1}-\pi^{T}\right)^{2}}\right]\left(\frac{1}{2}-\lambda_{t-1}\right)$.

Com base em Gale, Binmore e Samuelson (1995), esse ruído pode ser acrescentado à dinâmica de seleção (10), originando a seguinte dinâmica evolucionária com taxa de mutação endógena:

$\lambda_{t}=\lambda_{t-1}+\left[1-\frac{\varepsilon \theta}{\varepsilon+\left(\pi_{t-1}-\pi^{T}\right)^{2}}\right]\left(1-\lambda_{t-1}\right)\left[1-\left(1+\lambda_{t-1}\right) F\left(\delta\left(\pi_{t-1}-\pi^{T}\right)^{2}\right)\right]+\left[\frac{\varepsilon \theta}{\varepsilon+\left(\pi_{t-1}-\pi^{T}\right)^{2}}\right]\left(\frac{1}{2}-\lambda_{t-1}\right)$.

Notamos que para $\theta=0$ a dinâmica evolucionária (13) torna-se a dinâmica de seleção (10).

A transição de estado do modelo é governada pelas equações em diferenças (6) e (13). Este sistema apresenta um único equilíbrio. Com efeito, com base em (6), inferimos que para qualquer $\lambda_{t-1}>0$, teremos $\pi_{t}-\pi^{T}=\pi_{t-1}-\pi^{T} \equiv \pi^{*}-\pi^{T}$ se, e somente se, $\pi^{*}-\pi^{T}=0$, ou seja, se, e somente se, a inflação de equilíbrio $\pi^{*}$ for igual à meta da inflação. Sob a condição de que o desvio da inflação observada com relação à meta é nulo, e considerando que $F(0)=0$, segue de (13) que a fração de crédulos de equilíbrio $\lambda^{*}$ deve satisfazer a seguinte condição:

$$
(1-\theta)\left(1-\lambda^{*}\right)+\theta\left(\frac{1}{2}-\lambda^{*}\right)=0
$$

cuja solução é $\lambda^{*}=1-\theta / 2 \in(0,1] \subset \mathbb{R}$ para qualquer $\theta \in[0,1) \subset \mathbb{R}$. Concluímos, então, que há um único equilíbrio, a saber, o equilíbrio $\left(\lambda^{*}, \pi^{*}-\pi^{T}\right)=(1-(\theta / 2), 0)$. Cabe salientar que o valor de equilíbrio do grau de credibilidade, dado por $\lambda^{*}$, varia negativamente com a taxa de mutação $\theta$. 
Neste estado de equilíbrio, temos $i_{t-1}-\pi_{t}^{e}=r_{n}$ por (3) e, portanto, $y_{t}=y_{n}$ por (1). Ademais, com base em (4), segue que $\pi_{t}^{e}=\pi_{t}=\pi^{T}$.

Em suma, o equilíbrio do sistema em análise é caracterizado por credibilidade incompleta $\left(\lambda_{t}<1\right)$ se $\theta>0$ e plena $\left(\lambda_{t}=1\right)$ se $\theta=0$, bem como por uma configuração macroeconômica na qual o produto encontra-se no seu nível natural $\left(y_{t}=y_{n}\right)$, as taxas de inflação esperada e observada são iguais à meta preestabelecida $\left(\pi_{t}^{e}=\pi_{t}=\pi^{T}\right) \mathrm{e}$ a taxa de juros nominal natural, $i_{n}$, está ancorada na meta de inflação e na taxa de juros real natural $\left(i_{t}=i_{n}=\pi^{T}+r_{n}\right)$. Para que a taxa nominal de juros no equilíbrio seja não negativa, devemos impor a restrição $\pi^{T}+r_{n} \geq 0$.

Esta última restrição deve, de fato, valer para qualquer período. Inserindo (5) em (3), e após algumas manipulações algébricas, podemos expressar a taxa de juros nominal como segue:

$i_{t-1}=\lambda_{t-1} \pi^{T}+\left(1-\lambda_{t-1}\right) \pi_{t}+r_{n}+\gamma\left(\pi_{t-1}-\pi^{T}\right)=i_{n}+\left(1-\lambda_{t-1}\right)\left(\pi_{t}-\pi^{T}\right)+\gamma\left(\pi_{t-1}-\pi^{T}\right)$.

Finalmente, inserindo (6) em (15), o limite inferior zero para a taxa nominal de juros valerá em um período $t$ qualquer se a seguinte condição for satisfeita:

$$
i_{n}+\gamma\left(1+\alpha \beta-\frac{\alpha \beta}{\lambda_{t}}\right)\left(\pi_{t}-\pi^{T}\right) \geq 0 .
$$

Considerando (16), o espaço de estados para o qual a taxa nominal de juros é positiva (i.e., não negativa) pode ser assim expresso:

$$
\Theta=\left\{\left(\lambda_{t}, \pi_{t}-\pi^{T}\right) \in \mathbb{R}^{2}: 0<\lambda_{t} \leq 1, i_{n}+\gamma\left(1+\alpha \beta-\frac{\alpha \beta}{\lambda_{t}}\right)\left(\pi_{t}-\pi^{T}\right) \geq 0\right\} .
$$

Com base na desigualdade (16), inferimos que a fronteira que delimita o espaço de estados economicamente relevante com base no sinal da taxa de juros nominal é dada por: 


$$
\pi_{t}-\pi^{T}=\frac{i_{n} / \gamma}{\frac{\alpha \beta}{\lambda_{t}}-(1+\alpha \beta)}, \text { para todo } \lambda_{t} \in(0,1] \subset \mathbb{R} \text { e } \lambda_{t} \neq \frac{\alpha \beta}{1+\alpha \beta} \equiv \bar{\lambda}
$$

Esta função apresenta uma descontinuidade em $\bar{\lambda}$, de maneira que a reta $\lambda_{t}=\bar{\lambda}$ é uma assíntota vertical do gráfico desta função, representado pelas curvas "traço-ponto-traço", que são fronteiras das regiões hachuradas da Figura 1. Estas regiões são formadas por estados nos quais a taxa de juros nominal é estritamente negativa. Portanto, a região não hachurada, incluindo as referidas fronteiras, representa o espaço de estados economicamente relevante $\Theta$.

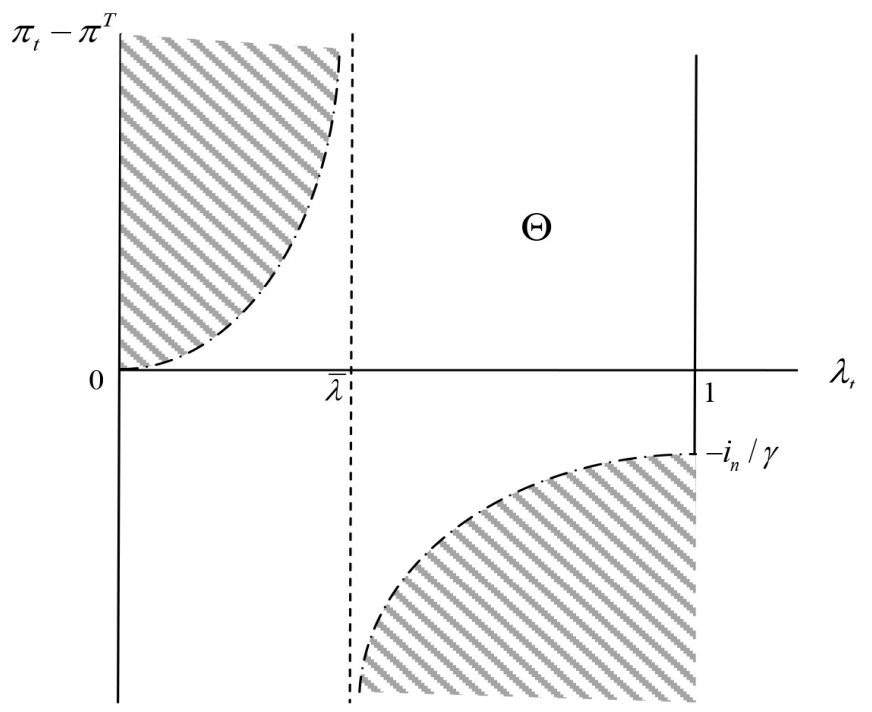

Figura 1. Espaço de estados economicamente relevante

Vejamos se o equilíbrio $\left(\lambda^{*}, \pi^{*}-\pi^{T}\right)=(1-(\theta / 2), 0)$ é um atrator da dinâmica definida pelas equações de transição de estado (6) e (13). Consideremos a matriz jacobiana da linearização em torno do equilíbrio em análise:

$$
J\left(\lambda^{*}, \pi^{*}-\pi^{T}\right)=\left[\begin{array}{c|c}
0 & 0 \\
\hline 0 & \frac{-\alpha \beta \gamma}{1-(\theta / 2)}
\end{array}\right],
$$


cujos autovalores são $\lambda_{1}=0$ e $\lambda_{2}=\frac{-\alpha \beta \gamma}{1-(\theta / 2)}$. Desde que $\alpha \beta \gamma>0$

e $0 \leq \theta<1$, teremos $\lambda_{2}<0$. Logo, o equilíbrio será um atrator local se $\frac{-\alpha \beta \gamma}{1-(\theta / 2)}>-1$, ou seja, se

$$
\alpha \beta \gamma<1-(\theta / 2) .
$$

Portanto, para dados valores dos parâmetros $\alpha$ e $\beta$, relativos ao comportamento do setor privado da economia, e da taxa de mutação $\theta$, a autoridade monetária não poderá reagir muito fortemente a desvios da inflação contemporaneamente observada com relação à meta. $\mathrm{Ou}$ seja, o parâmetro $\gamma$ deve ser suficientemente reduzido para que a condição de estabilidade (20) tenha validade. Além disso, é interessante observar que a reação da autoridade monetária a um desvio da inflação contemporaneamente observada com relação à meta deverá ser tanto mais reduzida quanto mais intensos forem os efeitos do hiato de produto a uma variação no hiato de taxa de juros, dado pelo parâmetro $\alpha$ na curva IS, e da taxa de inflação a uma variação contemporânea no hiato de produto, dado pelo parâmetro $\beta$ na curva de Phillips. Sob tais condições, a economia convergirá para seu equilíbrio macroeconômico com o máximo de credibilidade possível caso se encontre na bacia de atração deste equilíbrio.

Logo, uma importante implicação do modelo aqui desenvolvido é que o princípio de Taylor não é uma condição necessária para a existência e estabilidade do equilíbrio. Esse princípio, inicialmente formulado em um modelo novo-Keynesiano por Woodford (2001) com base na contribuição de Taylor (1993), estipula que a taxa de juros nominal deve reagir positivamente, mas mais que proporcionalmente, a uma variação na taxa de inflação. No caso do presente modelo, a resposta contemporânea da taxa de juros nominal a uma elevação na taxa de inflação é dada pelo parâmetro $\gamma$, o qual, porém, como visto acima, não precisa ser maior que a unidade (o que caracterizaria a satisfação do princípio de Taylor) para que o equilíbrio com alcance da meta de inflação seja estável. A razão dessa independência da estabilidade do equilíbrio em relação ao princípio de Taylor resulta da natureza voltada para frente (forward looking) da regra de juros representada por (3), de acordo com a qual a taxa de juros nominal reage contemporaneamente não apenas ao hiato de inflação, mas, inclusive, à 
taxa de inflação esperada. Esta última, por sua vez, conforme (5), é formada, em parte, pela estimativa dos agentes céticos quanto ao alcance da meta de inflação, os quais formam suas expectativas de forma plenamente racional, pagando um custo para prever com perfeição a taxa de inflação no período seguinte. É interessante notar, portanto, que esse ceticismo, em última instância, é funcional para a autoridade monetária: ao anteciparem perfeitamente um aumento na taxa de inflação no período seguinte, os agentes céticos induzem a autoridade monetária a elevar a taxa de juros nominal já no período corrente. E, conforme (1), a elevação na taxa de juros real esperada daí resultante reduz imediatamente a demanda agregada. Em outras palavras, os agentes privados céticos, para quem a autoridade monetária não é crível o suficiente, contribuem para que a política monetária seja, em parte, preventiva.

No entanto, mesmo valendo a condição de estabilidade (20), haverá oscilação da inflação observada em torno da meta de inflação, caso a primeira seja diferente da última. Com efeito, para $\pi_{t-1}-\pi^{T}>0$, haverá queda da inflação no próximo período, a qual ficará abaixo da meta, pois de (6) temos $\Delta \pi_{t} \equiv \pi_{t}-\pi_{t-1}=\pi_{t}-\pi^{T}-\left(\pi_{t-1}-\pi^{T}\right)<0$. Por sua vez, haverá aumento da inflação e esta ficará acima da meta em $t$ se $\pi_{t-1}-\pi^{T}<0$. A oscilação da inflação observada em torno da meta será amortizada ou amplificada a depender da magnitude do fator multiplicativo $-\alpha \beta \gamma / \lambda_{t-1}$ que aparece em (6).

A título de ilustração, apresentamos na Figura 2 o diagrama de fases para o caso sem mutação $(\theta=0)$. Com base na argumentação desenvolvida no parágrafo anterior, inferimos que as setas direcionais associadas à variável $\pi_{t}-\pi^{T}$ apontam para baixo em estados nos quais a inflação observada está acima da meta de inflação, enquanto apontam para cima quando a primeira é superada pela última. Somente no caso em que $\pi_{t}-\pi^{T}=0$ é que o ajustamento do grau de credibilidade não é acompanhado pela oscilação da inflação observada em torno da meta.

Por sua vez, as fronteiras representadas pelas curvas "traço-ponto-ponto-traço" são loci de estados para os quais $\Delta \lambda_{t}=\lambda_{t}-\lambda_{t-1}=0$, obtidas como funções implícitas da condição ${ }^{5} 1-\left(1+\lambda_{t-1}\right) F\left(\delta\left(\pi_{t-1}-\pi^{T}\right)^{2}\right)=0$, a qual pode ser reescrita para um período $t$ qualquer como segue:

${ }^{5}$ O lado esquerdo da igualdade adiante é a expressão entre colchetes na equação em diferenças (10). 


$$
F\left(\delta\left(\pi_{t}-\pi^{T}\right)^{2}\right)=\frac{1}{1+\lambda_{t}}
$$

Desde que $F^{\prime}(\cdot)>0$ em todo seu domínio, segue que $\left(\pi_{t}-\pi^{T}\right)^{2}$ é uma função estritamente decrescente com relação a $\lambda_{t}$, pois

$$
\frac{(\pi \pi)}{d \lambda_{t}}=\frac{}{\left(1+\lambda_{t}\right) \delta F^{\prime}(\cdot)}<\quad \text { para todo } \lambda_{t} \in(0,1] \subset \mathbb{R} \text {. }
$$

Ademais, se $\lambda_{t} \rightarrow 0^{+}$segue que $F(\cdot) \rightarrow 1^{-}$e, portanto, $\left(\pi_{t}-\pi^{T}\right)^{2} \rightarrow+\infty$. Por sua vez, se $\lambda_{t} \rightarrow 1^{-}$segue que $F(\cdot) \rightarrow(1 / 2)^{-}$e, consequentemente, $\left(\pi_{t}-\pi^{T}\right)^{2^{t}} \rightarrow\left(\bar{\pi}-\pi^{T}\right)^{2}$, sendo $\bar{\pi}$ o valor da inflação tal que $F\left(\delta\left(\bar{\pi}-\pi^{T}\right)^{2}\right)=1 / 2$. Em suma, estas propriedades associadas à função implícita (21) permitem traçar os gráficos das curvas de demarcação $\Delta \lambda_{t}=\lambda_{t}-\lambda_{t-1}=0$ apresentados na Figura 2.

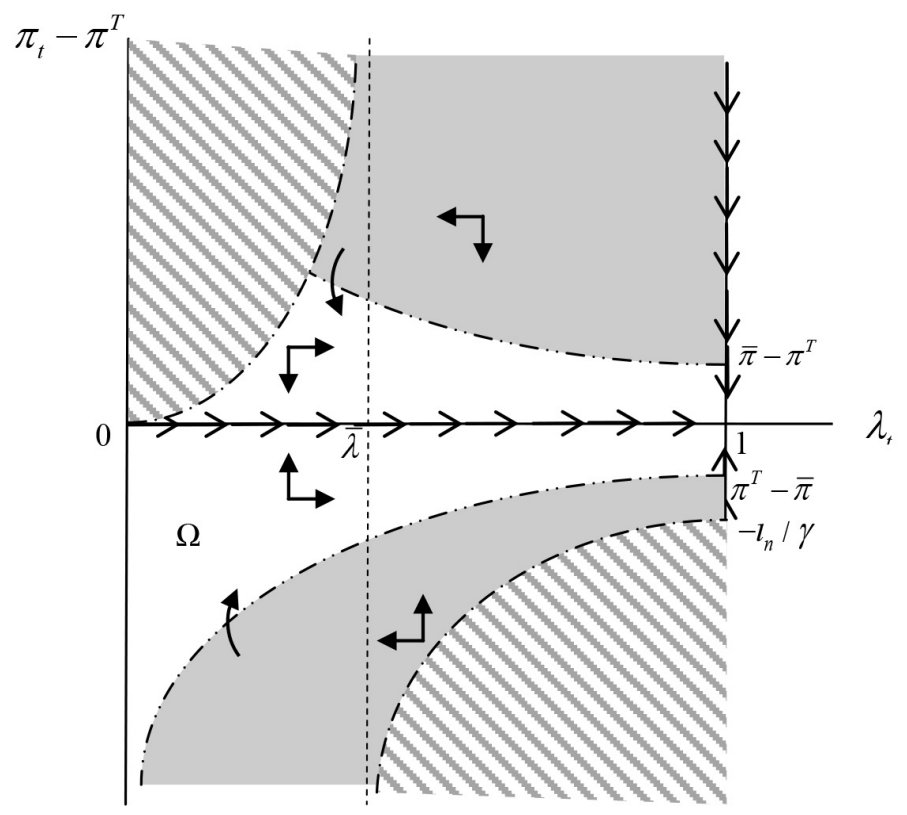

Figura 2. Digrama de fases para $\theta=0$ 
Consideremos a região destacada em cor branca no diagrama de fases da Figura 2, formada pelos estados pertencentes ao seguinte conjunto:

$$
\Omega \equiv\left\{\left(\pi_{t-1}-\pi^{T}, \lambda_{t-1}\right) \in \Theta: 1-\left(1+\lambda_{t-1}\right) F\left(\delta\left(\pi_{t-1}-\pi^{T}\right)^{2}\right) \geq 0\right\} .
$$

Podemos inferir que, para um dado estado de credibilidade $\lambda_{t-1}$, se o desvio da inflação com relação à meta for suficientemente baixo, no sentido de que $\left(\lambda_{t}, \pi_{t}-\pi^{T}\right) \in \Omega$, a estratégia crédula torna-se mais atrativa e, portanto, a medida de crédulos tende a aumentar $\left(\Delta \lambda_{t}>0\right)$. O oposto ocorrerá se o desvio da inflação com relação à meta for demasiadamente grande. Em suma, fora (dentro) da região $\Omega$ a seta direcional associada à variável $\lambda_{t}$ aponta para esquerda (direita). Portanto, em uma situação de queda do grau de credibilidade, se a autoridade monetária reagir moderadamente a um desvio da inflação contemporaneamente observada com relação à meta, reduzindo assim a amplitude do desvio da primeira em relação à última, a tendência de retrocesso da credibilidade será revertida, pois a perda relativa da estratégia crédula diminui, atraindo assim novos agentes para esta estratégia.

\section{Considerações Finais}

Investigamos a dinâmica de um regime monetário de meta de inflação em um contexto macroeconômico representado por um modelo de três equações (curva IS, curva de Phillips e regra de juros) e com heterogeneidade de estratégias de formação de expectativas de inflação. Os agentes econômicos podem optar por usar a meta de inflação anunciada como previsor da inflação futura (estratégia crédula) ou pagar um custo aleatório (e desconhecido a priori) para prever com perfeição esta inflação (estratégia cética).

A evolução da distribuição de estratégias de formação de expectativas foi modelada como um processo evolucionário, composto por duas forças: seleção e mutação. Enquanto a primeira reflete a atratividade relativa das estratégias em termos de payoffs, a segunda reflete escolhas aleatórias desconectadas dos payoffs. 
A dinâmica de coevolução da macroeconomia e da distribuição de estratégias de formação de expectativas apresenta um único equilíbrio, caracterizado por credibilidade incompleta, se há perturbação advinda de mutação, e credibilidade plena, no caso contrário. Do ponto de vista macroeconômico, porém, em ambos os casos o produto converge para o seu nível natural $\left(y_{t}=y_{n}\right)$, as taxas de inflação esperada e observada tornam-se iguais à meta preestabelecida pela autoridade monetária e a taxa nominal de juros acaba ancorada na meta de inflação e na taxa de juros real natural. Logo, em termos de gestão monetária, uma implicação relevante do modelo desenvolvido é que a credibilidade plena não é uma condição necessária para o alcance da meta de inflação, já que a heterogeneidade de expectativas de inflação, na presença de mutação e com alcance da meta de inflação, é um equilíbrio evolucionário.

Uma condição importante para que haja convergência para o equilíbrio com alcance da meta de inflação é a de que a autoridade monetária não reaja muito fortemente a desvios da inflação contemporaneamente observada com relação à meta. Sob tal condição, embora a autoridade monetária produza oscilação da inflação em torno da meta durante a dinâmica de transição para o equilíbrio, a economia convergirá para seu equilíbrio macroeconômico com o máximo de credibilidade possível. Sendo assim, uma importante implicação do modelo é que o princípio de Taylor não é uma condição necessária para a estabilidade do equilíbrio. A razão é que a regra de juros que guia a política monetária é voltada para frente (forward looking), com a taxa de juros nominal reagindo contemporaneamente não apenas ao hiato de inflação, mas, inclusive, à taxa de inflação esperada. Esta última, por sua vez, é formada, em parte, pela estimativa dos agentes que, por serem céticos quanto ao alcance da meta de inflação, formam suas expectativas de forma plenamente racional, pagando um custo para prever com perfeição a taxa de inflação no período seguinte. Logo, esse ceticismo, em última instância, é funcional para a autoridade monetária: ao anteciparem perfeitamente um aumento na taxa de inflação no período seguinte, os agentes céticos induzem a autoridade monetária a elevar a taxa de juros nominal já no período corrente. Ou seja, os agentes privados céticos, para quem a autoridade monetária não é crível o suficiente, contribuem para que a política monetária seja, ao menos em parte, devidamente preventiva. 


\section{Referências}

ADAM, K. Experimental evidence on the persistence of output and inflation, The Economic Journal, 117(520), 603-636, 2007.

ASSENZA, T., Heemeijer, P., Hommes, C. e Massaro, D. Individual expectations and aggregate macro behavior, DNB Working Paper, n. 298, May, 2011.

Banco Central do Brasil. Relatório de inflação, 14(1), Março, 2012.

BEVILAQUA, A.; Mesquita, M.; Minella, A. Brazil: taming inflation expectations, Working Paper Series, n. 129, Banco Central do Brasil, 2007.

BRANCH, W. A. The theory of rationally heterogeneous expectations: evidence from survey data on inflation expectations, The Economic Journal, 114(497), p. 592-621, 2004.

. Sticky information and model uncertainty in survey data on inflation expectations, Journal of Economic Dynamics and Control, 31 (1), p. 245-276, 2007.

BRANCH, W. A.; McGough, B. Monetary policy in a new Keynesian model with heterogeneous expectations. Journal of Economic Dynamics and Control, 33 (5), p. 1036-1051, 2009.

Dynamic Predictors Selection in a New Keynesian Model with Heterogeneous Expectations, Journal of Economic Dynamics and Control, 34(8), p. 1492-1508, 2010.

BRAZIER, A.; Harrison, R.; King, M.; Yates, T. The danger of inflating expectations of macroeconomic stability: heuristic switching in an overlapping generations monetary model, International Journal of Central Banking, 4, p. 219-254, 2008.

BROCK, W. A.; Hommes, C. H. A Rational route to randomness, Econometrica, v. 65, p. 1059-1160, 1997.

CALVO, G. Staggered prices in a utility maximizing framework, Journal of Monetary Economics, 12 , p. 383-398, 1983.

CAPISTRÁN, C.; Timmermann, A. Disagreement and biases in inflation expectations, Journal of Money, Credit and Banking, 41, p. 365-396, 2009.

CARROLL, C. D. Macroeconomic expectations of households and professional forecasters. Quarterly Journal of Economics, 118 (1), p. 269-298, 2003.

The epidemiology of macroeconomic expectations. In: Blume, L.; Durlauf, S. (eds). The Economy as an Evolving Complex System, III, Oxford: Oxford University Press, 2006.

CERISOLA, M.; Gelos, R. G. What drives inflation expectations in Brazil? An empirical analysis, IMF Working Paper, n. 05/109, Washington, DC, 2005.

CONLISK, J. Why bounded rationality?, Journal of Economic Literature, Vol. XXXIV, p. 669-700, 1996.

DE GRAUWE, P. Animal Spirits and Monetary Policy, Economic Theory, p. 1-35, 2010.

DIRON, M.; Mojon, B. Forecasting the central bank's inflation objective is a good rule of thumb, $E C B$ Working Paper, n. 564, 2005.

DUFFY, J. Experimental macroeconomics. In: Durlauf, S.; Blume, and L. (eds.). New Palgrave Dictionary of Economics. New York: Palgrave Macmillan, 2008.

EVANS, G. W; Honkapohja, S. Learning and expectations in macroeconomics, Princeton: Princeton University Press, 2001.

GALE, J.; Binmore, K.; Samuelson, L. Learning to be imperfect: the ultimatum game, Games and Economic Behavior, 8(1), p. 56-90, 1995.

GALÍ, J. Monetary policy, inflation and the business cycle: an introduction to the new keynesian framework. Princeton: Princeton University Press, 2008.

HOMMES, C. H. The heterogeneous expectations hypothesis: some evidence from the lab, Journal of Economic Dynamics and Control, 35, p. 1-24, 2011. 
HOMMES, C. H.; Sonnemans, J.; Tuinstra, J.; van de Velden, H. Coordination of expectations in asset pricing experiments, Review of Financial Studies, 18(3), p. 955-980, 2005.

KAPADIA, S. Inflation target expectations and optimal monetary policy, Oxford University Department of Economics Working Paper, n. 227, 2005.

LIMA, G. T.; Silveira, J. J. Nominal adjustment regimes in an evolutionary macrodynamics, Brazilian Review of Econometrics, v. 28, n. 1, p. 51-75, 2008.

MANKIW, N.; Reis, R. Sticky information versus sticky prices: a proposal to replace the New Keynesian Phillips curve, Quarterly Journal of Economics, 117(4), p. 1295-1328, 2002.

MANKIW, N. G.; Reis, R.; Wolfers, J. Disagreement about inflation expectations. In: NBER MacroECONOMICS ANNUAL 2003, V. 18, P. 209-270, 2004.

MANSKI, C.F.; McFadden, D. Structural analysis of discrete data with econometric applications, Cambridge, MA: MIT Press, 1981.

MARCET, A.; Sargent, T. J. Convergence of least squares learning mechanisms in self referential linear stochastic models, Journal of Economic Theory, 48(2), p. 337-368, 1989.

ORPHANIDES, A.; Williams, J.C. Robust monetary policy with imperfect knowledge, Journal of Monetary Economics, 54, p. 1406-1435, 2007.

PFAJFAR, D.; Santoro, E. Heterogeneity, learning and inflation stickiness in inflation expectations, Journal of Economic Behavior and Organization, 75(3), p. 426-444, 2010.

PFAJFAR, D.; Zakelj, B. Inflation expectations and monetary policy design: evidence from the laboratory," mimeo, Tilburg University, 2010.

SAMUELSON, L. Evolutionary games and equilibrium selection, Cambridge, MA: The MIT Press, 1997.

SILVEIRA, J. J.; Lima, G. T. Racionalidade limitada e neutralidade monetária: uma abordagem evolucionária, Revista Economia (ANPEC), v. 9, n. 4, p. 127-149, 2008.

TAYLOR, J. Discretion versus policy rules in practice, Carnegie-Rochester Conference Series on Public Policy, 39(1), pp. 195-214, 1993.

WEBER, A. Heterogeneous expectations, learning and European inflation dynamics, mimeo, 2010.

WOODFORD, M. The Taylor Rule and Optimal Monetary Policy, American Economic Review, Papers and Proceedings, v. 91, n. 2, p. 232-237, May 2001.

WOODFORD, M. Interest and prices, Princeton: Princeton University Press, 2003.

Imperfect common knowledge and the effects of monetary policy, in P. Aghion; Frydman, R.; Stiglitz, J.; Woodford, M. (eds) Knowledge, information and expectations in modern macroeconomics: essays in honor of Edmund Phelps, Princeton: Princeton University Press, p. 25-58, 2003. 